\title{
Derivation of Rotational Transformation Matrices via Rotational Invariant
}

\author{
Xiao-Ping Qin ${ }^{1,2}$, Peng $\mathrm{Li}^{1,2, a^{*}}$, Gang $\mathrm{Su}^{1,2}$, Ling-Mei Cong ${ }^{1,2}$ \\ ${ }^{1}$ School of Mathematics, Physics and Information Science, Zhejiang Ocean University, Zhoushan 316022, China \\ ${ }^{2}$ Key Laboratory of Oceanographic Big Data Mining \& Application of Zhejiang Province, Zhoushan 316022, China \\ apengli.physics@qq.com
}

\begin{abstract}
We derive the space rotational transformation matrices under two assumptions: the invariance of vector length and the allowance of infinitesimal rotation. The derivation is simple and concise by using trigonometric functions and the result is compared with previous studies. The method can be further generalized to derive the Lorentz transformation in spacetime.
\end{abstract}

Keywords-Vector; Rotational Transformation; Scalar

\section{INTRODUCTION}

In previous series we have comprehensively discussed the origin and applications of pseudovectors in physics [1-4]. In those studies most efforts were spent on the space rotation of vectors. Because it is crucial for a physical vector to abide the proper rotational transformation rule or it can't be considered as a proper vector. Such transformation rule is mathematically represented by the rotational matrices as shown in reference [1-4]. Now we raise a deeper question: how the rotation transformation matrices are obtained, or, what is the principle behind the construction of the rotation matrices?

In this paper we answer this question in detail. The basic idea is based on two facts: (i) the length of a vector should be invariant during the rotation, and (ii) the rotation must allow infinitesimal operation. We first show how these two principles lead to the well-defined rotation matrix, then apply this principle to the pseudovectors we studied before. It is seen that to justify whether a quantity is a vector, it is usually much simpler to verify whether its 'length' is a scalar than whether its coordinates transform according to the rotation matrix.

\section{LENGTH INVARIANCE AND ROTATIONAL TRANSFORMATION}

First we look at a rotation about the $z$ axis, such that only the $x$ and $y$ coordinates transform under rotation. In this case the position vector $\boldsymbol{r}$ transforms to $\boldsymbol{r}$ ' with the $z$ component untouched

$$
\boldsymbol{r}=(x, y, z) \rightarrow \boldsymbol{r}^{\prime}=\left(x^{\prime}, y^{\prime}, z\right)
$$

Now the invariance of length refers to $|\boldsymbol{r}|=\mid \boldsymbol{r} '$, i.e.,

This work is supported by the Teaching Enhancement Program and the Doctoral Research Startup Fund of Zhejiang Ocean University, the Zhejiang Provincial Office of Education Grant No. Y201534406, and NSFC Grant No 11505154, 11305141. *Author to whom correspondence should be addressed.

$$
x^{2}+y^{2} \equiv\left(x^{\prime}\right)^{2}+\left(y^{\prime}\right)^{2}
$$

We now seek a transformation that always preserves the equality (2).

Provided the transformation being linear and homogeneous, it can be tentatively written as

$$
\left\{\begin{array}{l}
x^{\prime}=a x+b y \\
y^{\prime}=c x+d y
\end{array}\right.
$$

where the coefficients $a, b, c$, and $d$ are to be determined. Inserting equation (3) into (2) we find

$$
\begin{aligned}
x^{2}+y^{2} & =(a x+b y)^{2}+(c x+d y)^{2} \\
& =\left(a^{2}+c^{2}\right) x^{2}+\left(b^{2}+d^{2}\right) y^{2}+2(a b+c d) x y
\end{aligned}
$$

By equating the corresponding terms of $x$ and $y$ we obtain the following set of equations

$$
\left\{\begin{array}{l}
a^{2}+c^{2}=1 \\
b^{2}+d^{2}=1 \\
a b+c d=0
\end{array}\right.
$$

The first equation in (5) suggest that $a$ and $c$ can be denoted by the trigonometric functions, without loss of generality, as

$$
\left\{\begin{array}{l}
a=\cos \theta \\
c=\sin \theta
\end{array}\right.
$$

where $\theta$ is an arbitrary parameter. Similarly, the second equation in (5) suggest that

$$
\left\{\begin{array}{l}
b=\sin \xi \\
d=\cos \xi
\end{array}\right.
$$


where $\xi$ is another arbitrary parameter. Note here we have changed the order of sine and cosine to facilitate latter derivations. Inserting (6) and (7) into the last equation of (5) we find

$$
\cos \theta \sin \xi+\sin \theta \cos \xi=0
$$

i.e.,

$$
\sin (\theta+\xi)=0
$$

Equation (9) has two distinct solutions, namely

$$
\theta+\xi=0
$$

and

$$
\theta+\xi=\pi
$$

It is straightforward to see that the first solution (10) leads to the following transformation

$$
\left\{\begin{array}{l}
x^{\prime}=x \cos \theta-y \sin \theta \\
y^{\prime}=x \sin \theta+y \cos \theta
\end{array}\right.
$$

The equations (12) are exactly the transformation of coordinates for a $z$-axis rotation of angle $\theta$. Meanwhile, the second solution (11) yields

$$
\left\{\begin{array}{l}
x^{\prime}=x \cos \theta+y \sin \theta \\
y^{\prime}=x \sin \theta-y \cos \theta
\end{array}\right.
$$

Although this transformation does preserve the length of vector $\boldsymbol{r}$, it is not the rotation transformation required in physics. The reason is apparent when infinitesimal transformation is considered, i.e., for $\theta \rightarrow 0$ it is clear that equation (13) reduces to

$$
\left(\begin{array}{l}
x \\
y
\end{array}\right) \rightarrow\left(\begin{array}{l}
x^{\prime} \\
y^{\prime}
\end{array}\right)=\left(\begin{array}{c}
x \\
-y
\end{array}\right)
$$

which is not the identity transformation. This is not an acceptable property of rotation, which must reduce to identity transformation at $\theta \rightarrow 0$. It is easy to verify that the transformation (12) does satisfy this requirement. As a conclusion, we assert that the transformation for $z$-axis rotation must have exactly the form as shown in equation (12).

The above analysis can be easily generalized to rotations about the $x$ and $y$ axes. By simply applying the cyclic rule,

$$
x \rightarrow y, y \rightarrow z \text {, and } z \rightarrow x
$$

to equation (13), we obtain the rotational transformation matrices about the three Cartesian axes as

$$
\begin{aligned}
& \boldsymbol{R}_{z, \theta}=\left(\begin{array}{ccc}
\cos \theta & -\sin \theta & 0 \\
\sin \theta & \cos \theta & 0 \\
0 & 0 & 1
\end{array}\right) \\
& \boldsymbol{R}_{x, \theta}=\left(\begin{array}{ccc}
1 & 0 & 0 \\
0 & \cos \theta & -\sin \theta \\
0 & \sin \theta & \cos \theta
\end{array}\right) \\
& \boldsymbol{R}_{y, \theta}=\left(\begin{array}{ccc}
\cos \theta & 0 & \sin \theta \\
0 & 1 & 0 \\
-\sin \theta & 0 & \cos \theta
\end{array}\right)
\end{aligned}
$$

Finally, the general rotation matrix $\boldsymbol{R}$ is an arbitrary combination (multiplications) of above matrices. The aggregation of all such rotational matrices forms a continuous group called the $\mathrm{SO}(3)$.

\section{SCALAR AND VECTORS IN PHYSICS}

Since the rotational transformation matrices (16) are deduced from length invariance, it is safe to say that if a quantity $\boldsymbol{A}=\left(A_{x}, A_{y}, A_{z}\right)$ preserves its length square

$$
|\boldsymbol{A}|^{2}=A_{x}^{2}+A_{y}^{2}+A_{z}^{2}
$$

under space rotation, then $\boldsymbol{A}$ must be a proper vector. Such criterion is equivalent to verifying whether $\boldsymbol{A}$ abides the rotation matrix $\boldsymbol{R}$. Generally, if a quantity is invariant under space rotation, it is called a scalar. Clearly, the length square of a vector is such a quantity.

Moreover, if $\boldsymbol{A}$ and $\boldsymbol{B}$ are two vectors, then the inner product

$$
\boldsymbol{A} \cdot \boldsymbol{B}=A_{x} B_{x}+A_{y} B_{y}+A_{z} B_{z}
$$

Is also a scalar. This can be easily shown as follows

$$
\begin{aligned}
\boldsymbol{A}^{\prime} \cdot \boldsymbol{B}^{\prime} & \equiv\left(\boldsymbol{A}^{\prime}\right)^{T} \boldsymbol{B}^{\prime}=(\boldsymbol{R} \boldsymbol{A})^{T}(\boldsymbol{R} \boldsymbol{B}) \\
& =\left(\boldsymbol{A}^{T} \boldsymbol{R}^{T}\right)(\boldsymbol{R} \boldsymbol{B})=\boldsymbol{A}^{T}\left(\boldsymbol{R}^{T} \boldsymbol{R}\right) \boldsymbol{B} \\
& =\boldsymbol{A}^{T} \boldsymbol{B}=\boldsymbol{A} \cdot \boldsymbol{B}
\end{aligned}
$$

where the vectors are treated as column matrices such that inner product can be calculated via matrix multiplication. Essentially, equation (19) holds because the rotational matrix $\boldsymbol{R}$ is orthogonal. 
On the other hand, if $\boldsymbol{A}$ is a vector and the quantity defined in (18) is a scalar, then $\boldsymbol{B}$ must be a vector. The proof is as follows. We assume under rotation $\boldsymbol{B}$ transforms to $\boldsymbol{B}$ ', and the transformation is linear and homogeneous such that it can be described by a matrix $\boldsymbol{S}$. Now if the inner product is invariant under rotation we must have the following equality

$$
\begin{aligned}
\left(\boldsymbol{A}^{\prime}\right)^{T} \boldsymbol{B}^{\prime} & =(\boldsymbol{R A})^{T}(\boldsymbol{S B})=\left(\boldsymbol{A}^{T} \boldsymbol{R}^{T}\right)(\boldsymbol{S} \boldsymbol{B}) \\
& =\boldsymbol{A}^{T}\left(\boldsymbol{R}^{T} \boldsymbol{S}\right) \boldsymbol{B}=\boldsymbol{A}^{T} \boldsymbol{B}
\end{aligned}
$$

Obviously the last step in equation (20) holds only if

$$
\boldsymbol{R}^{T} \boldsymbol{S}=\boldsymbol{I} \text {, i.e., } \boldsymbol{S}=\boldsymbol{R}
$$

because $\boldsymbol{R}$ is an orthogonal matrix. That is to say, $\boldsymbol{B}$ transforms under the rotational matrix $\boldsymbol{R}$, i.e., it must be a proper vector.

Next we use these criteria to verify several fundamental vector quantities in physics. The infinitesimal displacement

$$
d \boldsymbol{r}=(d x, d y, d z)
$$

is a vector because it is the difference between two position vectors $\boldsymbol{r}$ and $\boldsymbol{r}$ '. The velocity

$$
\boldsymbol{v}=\frac{d \boldsymbol{r}}{d t}=\left(\frac{d x}{d t}, \frac{d y}{d t}, \frac{d z}{d t}\right)
$$

is a vector because it is the vector $d \boldsymbol{r}$ divided by the scalar $d t$. Likewise, the acceleration

$$
\boldsymbol{a}=\frac{d \boldsymbol{v}}{d t}=\left(\frac{d v_{x}}{d t}, \frac{d v_{y}}{d t}, \frac{d v_{z}}{d t}\right)
$$

is a vector because it is the vector $d v$ divided by the scalar $d t$. The force obtained from the Newton's law

$$
\boldsymbol{F}=m \boldsymbol{a}
$$

is a vector because it is the vector $\boldsymbol{a}$ multiplied by a scalar $m$. A conservative force can be described otherwise as the gradient of a scalar potential field $\varphi(x, y, z)$, i.e.,

$$
\boldsymbol{F} \equiv-\nabla \varphi=-\left(\frac{\partial \varphi}{\partial x}, \frac{\partial \varphi}{\partial y}, \frac{\partial \varphi}{\partial z}\right)
$$

Because the potential $\varphi$ itself is a scalar, its infinitesimal increment $d \varphi$ along infinitesimal displacement $d \boldsymbol{r}$ is still a scalar,

$$
d \varphi=\nabla \varphi \cdot d \boldsymbol{r}=\frac{\partial \varphi}{\partial x} d x+\frac{\partial \varphi}{\partial y} d y+\frac{\partial \varphi}{\partial z} d z
$$

Because here $d \boldsymbol{r}$ is a vector, the gradient of a scalar field $\nabla \varphi$ must be a vector, so does the force defined in (26).

Now we look at a pseudovector, namely the torque $\boldsymbol{M}=\boldsymbol{r} \times \boldsymbol{F}$. We tentatively calculate its inner product with the vector $\boldsymbol{r}$, i.e.,

$$
\begin{aligned}
\boldsymbol{r} \cdot \boldsymbol{M} & =x\left(y F_{z}-z F_{y}\right)+y\left(z F_{x}-x F_{z}\right)+z\left(x F_{y}-y F_{x}\right) \\
& =0
\end{aligned}
$$

which is clearly a scalar, therefore the torque $\boldsymbol{M}$ must be vector. The cross product of any two vectors can be proved to be a vector in exactly the same way. Evidently, the proof (28) is exceedingly simple and concise than the method using transformation matrices as we have shown in [1].

Another kind of pseudovector is the curl of a vector field. Using the Stokes' theorem and let the trajectory $L$, as well as the surface element $S$ enclosed by it, shrinks to infinitesimal size, we obtain

$$
\lim _{\boldsymbol{S} \rightarrow 0}(\nabla \times \boldsymbol{A}) \cdot \boldsymbol{S}=\oint_{L \rightarrow 0} \boldsymbol{A} \cdot \boldsymbol{d l}
$$

The right hand term is an inner product so that it is a scalar. Because the surface element $\boldsymbol{S}$ itself is a vector, equation (29) suffices to speak that the curl of $\boldsymbol{A}$ is a vector. Here the proof (29) is also much simpler than what we have shown in Reference [3]. Interestingly, equation (29) can be used to define a trajectory independent potential provide that $\boldsymbol{A}$ is carless, and its applications can be found from here to there even in thermodynamics [5].

\section{CONCLUSION}

In this paper we have investigated the space rotation transformation in detail. The rotational transformation matrices are derived in a clear and simple approach by assuming length invariance. The rotational invariance of scalars and covariance of vectors are discussed with physical examples. We hope this paper helps to understand the mathematical structure of space rotation, which is a prevailing type of motion in both fundamental physics and mechanical engineering. Furthermore, the method can be easily generalized to derive the Lorentz transformation under fourdimensional spacetime and will be discussed in a subsequent paper [6].

\section{REFERENCES}

[1] Xiao-Ping Qin, Peng Li, Hong-Xia Wang and Zhao-Cun Zong, "Physical origin of pseudovector exemplified by torque", submitted to the International Conference on Arts and Design, Education and Social Sciences (ADESS 2017,Yinchuan), Atlantis Press. 
[2] Xiao-Ping Qin, Peng Li, Hong-Xia Wang and Zhao-Cun Zong, “Angular momentum, magnetic dipole moment, and classical picture of gyromagnetic ratio", submitted to the International Conference on Arts and Design, Education and Social Sciences (ADESS 2017, Yinchuan), Atlantis Press.

[3] Peng Li, Xiao-Ping Qin, Xue-Ping Cheng and Sheng-Wen Qi, "Curl of vector field as pseudovector", the 2nd International Conference on Automation, Mechanical Control and Computational Engineering (AMCCE 2017, Beijing), Atlantis Press.

[4] Peng Li, Xiao-Ping Qin, Xue-Ping Cheng and Sheng-Wen Qi, “Angular velocity, magnetic field, and gauge invariance", the 2nd International Conference on Automation, Mechanical Control and Computational Engineering (AMCCE 2017, Beijing), Atlantis Press.

[5] Li, Peng, Su Gang, Qin Xiao-Ping, “A Systematic Review of Classical Entropy", Proceedings of The 2016 International Forum on Management, Education and Information Technology Application (IFMEITA2016, Guangzhou), 47: 355-360. Atlantis Press

[6] Xiao-Ping Qin, Peng Li, Gang Su and Ling-Mei Cong, "Derivation of Lorentz Transformation via Lorentz Invariant”, submitted to 2nd International Conference on Automatic Control and Information Engineering (ICACIE 2017, Hongkong), Atlantis Press. 